sequently grow. Alternatively the shish kebab could be a development of the stacks of polymer crystals formed from static solutions, where the stacks resemble either an open book or a spiral staircase. If the gaps between crystals were opened up these would look like shish kebabs. Nagasawa and co-workers (IUPAC Symposium on Macromolecules, Aberdeen, 1973) reported the formation of shish kebabs by deformation of single crystal stacks and suggest that the shish kebab structures produced from flowing solutions are also the product of the mechanical disruption of stacked single crystals by the fluid flow. This idea would explain the similarity of structures produced under different conditions, the amount of connecting material between kebabs and the general irregularities of shish kebabs. This question could be resolved by studies of how shish kebabs grow.

Thus this elegant work will add to our understanding of flow in polymer solutions and thermodynamic aspects of crystallisation but tells us little about the crysallisation process. Methods are still needed to study the molecular arrangement in these structures. Results on crystallisation of polymer melts in the same apparatus should be as interesting and more directly relevant to polymer processing methods such as fibre spinning. In this respect this simple, controllable crystallisation system could be very enlightening.

\section{Halley lecturer produces new theory of comet origins}

\section{by John Gribbin}

ON May 6 the 1975 Halley Lecture was presented in Oxford by W. $\mathrm{H}$. McCrea, Professor Emeritus of the University of Sussex. McCrea took as the title of his discourse "The Solar System as a Space Probe", a far-ranging topic which suggests both an explanation of ice ages and a model for the origin of comets.

As McCrea pointed out, the advent of the space age has renewed interest in the study of the Solar System, but in many ways the Solar System itself is the best space probe of all, travelling at present towards a point in the constellation Hercules. The speed of the Solar System - $20 \mathrm{~km} \mathrm{~s}^{-1}$ relative to the rest frame of local stars; $140 \mathrm{~km} \mathrm{~s}^{-1}$ relative to the so-called 'high velocity' stars - may seem small compared with the speeds that are necessary to cross the Galaxy in a reasonable time, by human standards. But the great age of the Solar System, some $5 \times 10^{9}$ years, suggests that even at these modest speeds the system has already orbited around the Galaxy 20 times. So there has been ample scope for samples to have been gathered from different regions of the Galaxy.

The most obvious features of spiral galaxies, like our own, are the spiral arms (made up of bright stars) and the associated dark lanes of gas and dust. Can the passages of the Solar System through these features have left traces for us to discover? McCrea suggests that they have. The spiral arms are thought to be a more or less permanent feature, with the dark lanes along their edges showing the formation of shock waves as clouds of gas run into the arms.

In our Galaxy, there are two main arms, and in round terms the time scales associated with the motion of the Solar System produce a passage across one arm every 100 Myr. It takes $10 \mathrm{Myr}$ to cross each arm, and $1 \mathrm{Myr}$ to cross the lane of shocked and compressed clouds at the edge of the arm. Now, the Sun is just entering the Orion arm, having recently passed through the compression lane which lies just inside that arm.

Following in illustrious footsteps (including those of $H$. Shapley in 1920 and 1949, F. Hoyle and R. Lyttleton in 1939) McCrea suggests that it is just such passages of the Solar System through the dark lanes which bring about ice ages. Details of this new variation on an old theme are shortly to be published in Nature (in our issue marking the 300th anniversary of the foundation of the Royal Greenwich Observatory).

But the astronomers among McCrea's audience really began to sit up and take notice when he moved on to discuss the problem of the existence of coinets. These enigmatic objects, with masses in the range $10^{16}-10^{18} \mathrm{~g}$, are the least massive members of the Solar System (except for small asteroids) but in many ways the most baffling. Have they always formed part of the Sun's family, or are they ephemeral visitors snatched from the depths of space? Most theorists seem to lean towards the former view; but McCrea has now come up with a model which might change that consensus.

The most popular concept of a comet (but not the only model) sees it as a 'dirty snowball' of water ice and silicates. As McCrea notes, this composition is much the same as the best available models of interstellar grains, which are now regarded as silicates covered by ice. So, if a ball of interstellar dust could be separated out from the gas, we would have something very much like the nucleus of a comet. The snag is that the presence of the gas will tend to keep the interstellar grains apart even in a situation where with no gas they would congregate under their mutual gravitational attractions.

McCrea gets round this by suggesting that dust can collapse independently of the gas if the mean free path of a gas molecule (relative to dust grain encounters) is longer than the minimum radius of a cloud of dust at the same density which would collapse if free from gas. Putting in the numbers, he comes up with a range of masses for the resulting aggregations, and these are just in the range of comet masses. For example, grains $0.25 \mu \mathrm{m}$ across will form aggregates with mass about $1.6 \times 10^{18} \mathrm{~g}$. But to set the whole process off, the gas-dust mixture must be compressed to densities rather greater than the typical density of matter in intestellar space.

Once again, this is where the interaction of the moving gas with the spiral arms comes into the picture.

'Normal' clouds, says McCrea, have densities of about $0.1-10$ molecules $\mathrm{cm}^{-3}$. Dense clouds have about $10^{5}-10^{7}$ molecules $\mathrm{cm}^{-3}$ over regions one or more parsecs across, and the compression lanes must be at least as dense as the dense clouds. When a normal cloud hits a compression region, the first product is likely to be a dense cloud. At successive further compressions as the cloud continues its journey around the Galaxy and across spiral arms first cometary nuclei, then stellar and planetary condensations and even clusters of main sequence stars could be formed, depending on the starting density of a particular cloud. On that picture, the comets which now form part of the Solar System are a by-product of the recent passage through a compression lane, captured by the Sun en route. It may be that we are lucky to see any comets at all, and that for most of the long history of the Solar System there have been no comets bound to the Sun. Those we have at present will soon be dispersed, or broken up by repeated passage into the inner Solar System and past the Sun.

All this is dramatic enough. But the implications of the quite profound changes on Earth which occur as the Solar System moves through different regions of the Galaxy go still further. These "cosmic seasons", as Shapley dubbed them, could well have influenced the development of life, which (in the form we know it) may depend not just on the distance of a planet from its star, but also on the distance of the star from the galactic centre. McCrea terminated his lecture with the comment that the effect of all these astronomical influences on life "can be very complicated". 